OPEN ACCESS

Edited by:

Takayuki Matsumoto, Hoshi University, Japan

Reviewed by: Masashi Mukohda,

Okayama University of Science, Japan

Daisuke Nakano,

Kagawa University, Japan

${ }^{*}$ Correspondence:

Xueliang Wang

wangsun13141314@126

Hao Jiang

haojiang@ouc.edu.cn

Guangli Yu

glyu@ouc.edu.cn

Specialty section:

This article was submitted to Cardiovascular and Smooth Muscle

Pharmacology,

a section of the journal

Frontiers in Pharmacology

Received: 20 December 2021

Accepted: 20 January 2022

Published: 23 February 2022

Citation:

Wang $X$, Wang Z, Liu D, Jiang H, Cai C, Li G and Yu G (2022) Canagliflozin

Prevents Lipid Accumulation,

Mitochondrial Dysfunction, and Gut Microbiota Dysbiosis in Mice With

Diabetic Cardiovascular Disease.

Front. Pharmacol. 13:839640.

doi: 10.3389/fphar.2022.839640

\section{Canagliflozin Prevents Lipid Accumulation, Mitochondrial Dysfunction, and Gut Microbiota Dysbiosis in Mice With Diabetic Cardiovascular Disease}

\author{
Xueliang Wang ${ }^{1,2 *}$, Zhe Wang ${ }^{1}$, Di Liu ${ }^{1}$, Hao Jiang ${ }^{1,3 *}$, Chao Cai ${ }^{1,3}$, Guoyun $L^{1,3}$ and \\ Guangli $Y u^{1,3 *}$
}

${ }^{1}$ Key Laboratory of Marine Drugs of Ministry of Education, Shandong Provincial Key Laboratory of Glycoscience and Glycotechnology, School of Medicine and Pharmacy, Ocean University of China, Qingdao, China, ${ }^{2}$ Precision Medicine Institute, The First Affiliated Hospital of Sun Yat-Sen University, Sun Yat-Sen University, Guangzhou, China, ${ }^{3}$ Laboratory for Marine Drugs and Bioproducts, Pilot National Laboratory for Marine Science and Technology, Qingdao, China

Type 2 diabetes mellitus (T2DM) is associated with cardiovascular disease (CVD) and sodium glucose cotransporter 2 inhibitors, as oral medications for T2DM treatment have shown the potential to improve vascular dysfunction. The aim of this study was to evaluate the ability of canagliflozin (Cana) to relieve CVD in T2DM mice and its possible action mechanism. Mice with diabetic CVD was conducted by a high-fat diet for 24 weeks, followed by oral gavaging with metformin $(200 \mathrm{mg} / \mathrm{kg} /$ day) or Cana $(50 \mathrm{mg} / \mathrm{kg} / \mathrm{day})$ for 6 weeks. The result demonstrated that Cana reduced serum lipid accumulation, and decreased the arteriosclerosis index and atherogenic index of plasma. In addition, Cana treatment reduced the circulating markers of inflammation. More importantly, Cana improved cardiac mitochondrial homeostasis and relieved oxidative stress. Moreover, Cana treatment alleviated the myocardial injury with decreasing levels of serous soluble cluster of differentiation 40 ligand and cardiac troponin I. Thus, cardiovascular abnormality was relieved by suppressing fibrosis and basement membrane thickening, while elevating the cluster of differentiation 31 expression level. Importantly, Cana increased the ratio of gut bacteria Firmicutes/Bacteroidetes and the relative abundance of Alistipes, Olsenella, and Alloprevotella, while it decreased the abundance of Mucispirillum, Helicobacter, and Proteobacteria at various taxonomic levels in mice with diabetic CVD. In short, Cana treatment altered the colonic microbiota composition close to the normal level, which was related with blood lipid, inflammation, and oxidative stress, and might play a vital role in CVD. In general, the improvements in the gut microbiota and myocardial mitochondrial homeostasis may represent the mechanism of Cana on CVD treatment.

Keywords: canagliflozin, mitochondrion, hypolipidemic, colonic microbiota, myocardial protection, diabetic cardiovascular disease 


\section{INTRODUCTION}

Individuals with type 2 diabetes mellitus (T2DM) are at high risk for macrovascular complications (such as cerebrovascular and cardiovascular) (DeFronzo et al., 2015). T2DM is associated with cardiovascular disease (CVD) characterized by increases in endothelial dysfunction and large artery stiffness (Figueiredo et al., 2012). Risk factors of CVD include hyperglycemia, dyslipidemia, insulin resistance (IR), obesity, hypertension, nonalcoholic fatty liver disease, and so on (Brindle et al., 2006; Stevens et al., 2016). Although there is a correlation between CVD event rates and magnitude of hyperglycemia (Gerstein et al., 2005; Fox et al., 2015), there remains some nondeterminacy as to whether antidiabetic medications can reduce CVD risk (Group, 2008; Skyler et al., 2009). Dyslipidemia, oxidative stress, and inflammation are vital factors in the development of diabetic CVD (Nishikawa and Araki, 2007; Braunwald, 2008; Tietge, 2014). It was demonstrated that high-fat diet (HFD)induced elevated cholesterol and low-density lipoprotein cholesterol (LDL-C) resulted in both ischemic heart diseases with or without inflammation (Varbo et al., 2013). Hyperlipidemia is an elevation of lipids in the bloodstream (Jain et al., 2007), which is a cause of atherosclerosis and atherosclerosis-associated conditions (Aronson and Rayfield, 2002). Moreover, mitochondria are the most important sources of reactive oxygen species (ROS), and mitochondrial dysfunction increases electron leak and ROS production (Kayama et al., 2015).

In recent years, sodium glucose cotransporter 2 inhibitors (SGLT2i) were introduced as T2DM treatment (Rosenwasser et al., 2013). In meta-analyses of clinical trials in T2DM, three types of SGLT2i, including canagliflozin (Cana), dapagliflozin (Dapa), and empagliflozin (Empa) could reduce fasting plasma glucose and hemoglobin A1c (HbA1c) (Liakos et al., 2014; Sun et al., 2014; Yang et al., 2014). Moreover, it has been reported that several SGLT2i showed notable beneficial effects on CVD unlike other classes of antidiabetic medications (Dziuba et al., 2014; Lee et al., 2018; Jiang et al., 2021; Tan et al., 2021; Xiang et al., 2021).

In recent years, associations between T2DM and altered gut microbiome composition have been reported (Forslund et al., 2015). Moreover, many research indicate that the gut microbiota has emerged as a vital regulator of metabolic diseases, including in cardiovascular function in T2DM (Boulangé et al., 2016; Vikram et al., 2016; Battson et al., 2017). It has been verified that Dapa induced subtle alterations in intestinal flora in T2DM mice (Lee et al., 2018).

Cana was recently developed as a type of SGLT2i to improve T2DM and related diseases in an insulin-independent manner (Zinman et al., 2016; Steven et al., 2017). It has been reported that only Cana marked activated AMP-activated protein kinase (AMPK), but not Dapa, or Empa, which indicated a potential additional effect of Cana compared with other SGLT2i (Hawley et al., 2016). In addition, Cana decreased serum leptin and interleukin-6 (IL-6), while it increased serum adiponectin and tumor necrosis factor alpha (TNF-alpha) in T2DM patients, which favorably impacted insulin sensitivity and CVD risk (Garvey et al., 2018). Recently, several clinical studies indicated that cardiovascular events were lower after Cana treatment in T2DM patients (Neal et al., 2017; Mahaffey et al., 2018; Perkovic et al., 2019; Tian et al., 2021). In addition, Cana significantly altered cecal microbiota composition in renal failure mice and decreased the levels of microbiota-derived uremic toxins in chronic kidney disease (Mishima et al., 2017).

Here, we evaluated the effects of Cana on attenuating CVD in mice with T2DM induced by HFD (Supplementary Figure S1). Fasting blood glucose (FBG) and HbA1c levels were markedly increased after HFD was fed for 24 weeks compared with the NCD-fed group (Supplementary Figure S2). Meanwhile, oral glucose tolerance test (OGTT) and intraperitoneal insulin tolerance test (IPITT) assays demonstrated that the HFD-fed significantly decreased glucose tolerance (Supplementary Figure S2). These results demonstrated that mice with T2DM was successfully induced. Meanwhile, metformin (Metf), as a firstline clinical drug for T2DM, revealed marked improvement in reduction of CVD events (Fung et al., 2015). Thus, Metf was selected as the positive control. With the above background, the purpose of our study was to assess the effects of Cana on hyperlipemia, systemic inflammation, oxidative stress, IR, myocardial mitochondria, and vascular basement membrane in T2DM mice, and the effects of Cana on the gut microbiota dysbiosis was also characterized.

\section{MATERIALS AND METHODS}

\section{Chemicals and materials}

Metf and Cana were purchased from Aladdin (Aladdin Technology Co., Shanghai, China) and Selleck Chemicals (Selleck Chemicals, Houston, TX, USA) separately. All other chemicals were purchased from Sigma-Aldrich (St. Louis, MO, USA) unless otherwise noted.

\section{Animals and experimental design}

The experimental protocol is shown in Supplementary Figure S1. Briefly, male C57BL/6J mice aged 6 weeks with weights of 20-22 g were obtained from the Charles River (Beijing, China). Mice were allowed to acclimatize in the laboratory environment for 1 week before the beginning of the experiment. All mice had free access to food and water. Then mice were randomly divided into four groups ( $n=10$ per group): normal-chow diet (NCD)fed group (control group) (D12450B, Research Diets Inc., New Brunswick, NJ, USA), HFD-fed group (model group) (D12492, Research Diets Inc.), HFD plus Metf group (Metf group), and HFD plus Cana group (Cana group). Throughout the establishment of T2DM mice model period of 24 weeks, most individuals with HFD feeding appeared to be significantly hyperglycemic compared with the NCD feeding mice, while a few mice maintained a relatively normal blood glucose level. Thus, we randomly selected six mice with hyperglycemia for the following treatment trial. The sample size of each group was calculated by the $\mathrm{G}^{\star}$ Power software. In a one-way ANOVA with an effect size of $f=0.7, \alpha$ of 0.05 , power level of $80 \%$, and group number of 4 , it was calculated that a number of six mice per group would be sufficient to reach statistical significance. After 
that, the Metf and the Cana groups were gavaged with Metf $(200 \mathrm{mg} / \mathrm{kg}$ dissolve in saline solution containing $0.025 \%$ Tween-20 and $0.5 \%$ carboxymethyl cellulose) or Cana $(50 \mathrm{mg} / \mathrm{kg}$ dissolve in saline solution containing $0.025 \%$ Tween-20 and $0.5 \%$ carboxymethyl cellulose) once a day for six more weeks, while the control group and the model group were given equal amounts of solvents as a control vehicle. The body weight was weighed weekly. At the end of the experiment, mice were fasted for $12 \mathrm{~h}$ and were euthanized by pentobarbital sodium (100 mg/kg, ip) (Arjinajarn et al., 2016; Thongnak et al., 2017; Jaikumkao et al., 2018).

\section{Automatic hematological analysis}

Blood samples were collected in $1.5-\mathrm{ml} \mathrm{K}_{3}$ EDTA-containing tubes (Sarstedt, Nümbrecht, Germany) via the eyeball method, then analyzed by an automated veterinary hematology analyzer (Mindray, Shenzhen, China).

\section{Tissue sampling and histological study}

Cardiac tissues were collected, then partial cardiac tissues were fixed in a $4 \%$ paraformaldehyde solution or $2.5 \%$ glutaraldehyde for $48 \mathrm{~h}$, while frozen tissues were used for Oil Red $\mathrm{O}$ staining. Then tissues were dehydrated and embedded, and cut into sections. The tissue sections were analyzed by Oil Red O, H\&E, or Masson's trichrome staining (Goldner, 1938; Fischer et al., 2008; Wang et al., 2016).

\section{Biochemical assays}

FBG was measured after fasting for $12 \mathrm{~h}$. Oral glucose tolerance test (OGTT) and intraperitoneal insulin tolerance test (IPITT) assays were conducted (Fraulob et al., 2010; Fei and Zhao, 2013). The increment of blood glucose after glucose loading was expressed in terms of area under the curve (AUC). Blood was collected and centrifuged at $375 \times g$ for $10 \mathrm{~min}$ to obtain serum. Hemoglobin A1c (HbAlc) and insulin in serum were determined using HbAlc and insulin ELISA Kits (OM549127 and OM451187; Omnimabs, Alhambra, CA, USA), respectively. Homeostasis model assessment-insulin resistance (HOMA-IR) was calculated as fasting insulin $\times$ fasting glucose $/ 22.5$. Serum triglyceride (TG), total cholesterol (TC), high-density lipoprotein cholesterol (HDL-C), and low-density lipoprotein cholesterol (LDL-C) levels were detected by assay kits (F001-1-1, F002-11, A112-1-1, and A113-1-1; Jiancheng Bioengineering Institute, Nanjing, China). IL-6, monocyte chemotactic protein 1 (MCP-1), and TNF-alpha levels were determined using ELISA kits (M6000B, 479-JE-010/CF, DY410-05; R\&D systems, Minneapolis, MN, USA). Soluble cluster of differentiation 40 ligand (sCD40L) and cardiac troponin I (cTn I) contents were determined using ELISA kit (OM448329 and OM453121; Omnimabs, Alhambra, CA, USA). Cardiac tissues (200 mg) were homogenized in cold phosphate buffer, then centrifuged to collect the supernatant. Glutathione (GSH), superoxide dismutase (SOD), malondialdehyde (MDA), and catalase (CAT) contents in cardiac tissue were determined (A006-2-1, A001-3-2, A003-4-1, and A007-1-1; Nanjing Jiancheng Bioengineering Institute, Nanjing, China). In addition, ROS content in cardiac tissue was detected by fluorescent dye dihydroethidium (DHE) as previously described (Zheng et al., 2018).

\section{Immunohistochemistry and immunofluorescence assays}

Cluster of differentiation 31 (CD31) (AF6191, Affinity Biosciences, Cincinnati, OH, USA) and heme oxygenase 1 (HO-1) (86806S, Cell Signaling Technology, Danvers, MA, USA) in cardiac tissues were determined as previously described (El-Azab et al., 2012). After blocking with goat serum, sections were incubated with primary antibody at $4^{\circ} \mathrm{C}$ for $12 \mathrm{~h}$ and then incubated at room temperature for $1 \mathrm{~h}$ with fluorescent secondary antibody. Then the sections were stained with DAPI for $30 \mathrm{~min}$, and images were acquired.

\section{TdT-mediated fluorescein nucleotide (cyanine 3-dUTP) nick-end labeling detection}

TdT-mediated fluorescein nucleotide (cyanine 3-dUTP) nick-end labeling (TUNEL) was conducted using the TUNEL Assay Kit (ab66110, Abcam, Cambridge, UK) to evaluate the apoptosis of cardiac tissues. the incorporated fluorescein-labeled dUTP was analyzed.

\section{Transmission electron microscope}

Transmission electron microscopy (TEM) of cardiac tissues was conducted as previously described (Das et al., 2017). Cardiac tissues were fixed in $2.5 \%$ glutaraldehyde for $48 \mathrm{~h}$ at room temperature. Tissues were embedded in araldite, then cardiac microvasculature and mitochondria were observed by an electron microscope. The vascular basement membrane thickness of each sample was determined by the average value of six measurements at various position.

\section{Colonic microbiota high-throughput sequencing}

Total bacterial DNA was isolated from colonic contents by the QIAamp DNA Stool Mini Kit (51604, Qiagen, Hilden, Germany). The colonic bacterial 16S rDNA gene was amplified by PCR, then the PCR products were purified by the QIAquick Gel Extraction Kit (28706) and sequenced by Realbio (Realbio Technology Co., Ltd., Shanghai, China). Linear discriminate analysis effect size (LEfSe) was used to characterize the significant differences in the abundance of microbial taxa between different treated groups. Heatmap was analyzed by $\mathrm{R}$ Cytoscape. In addition, $\beta$-diversity was analyzed by the QIIME software.

\section{Western blot analysis}

Western blot analysis was conducted as in our previous study (Wang et al., 2021). In addition, the membranes were incubated with primary antibodies directed against pAMPK (2,531), AMPKa $(2,532)$, and $\beta$-actin $(4,970)$ from Cell Signaling Technology (Danvers, MA, USA). The ImageJ software was used for densitometry analysis. 

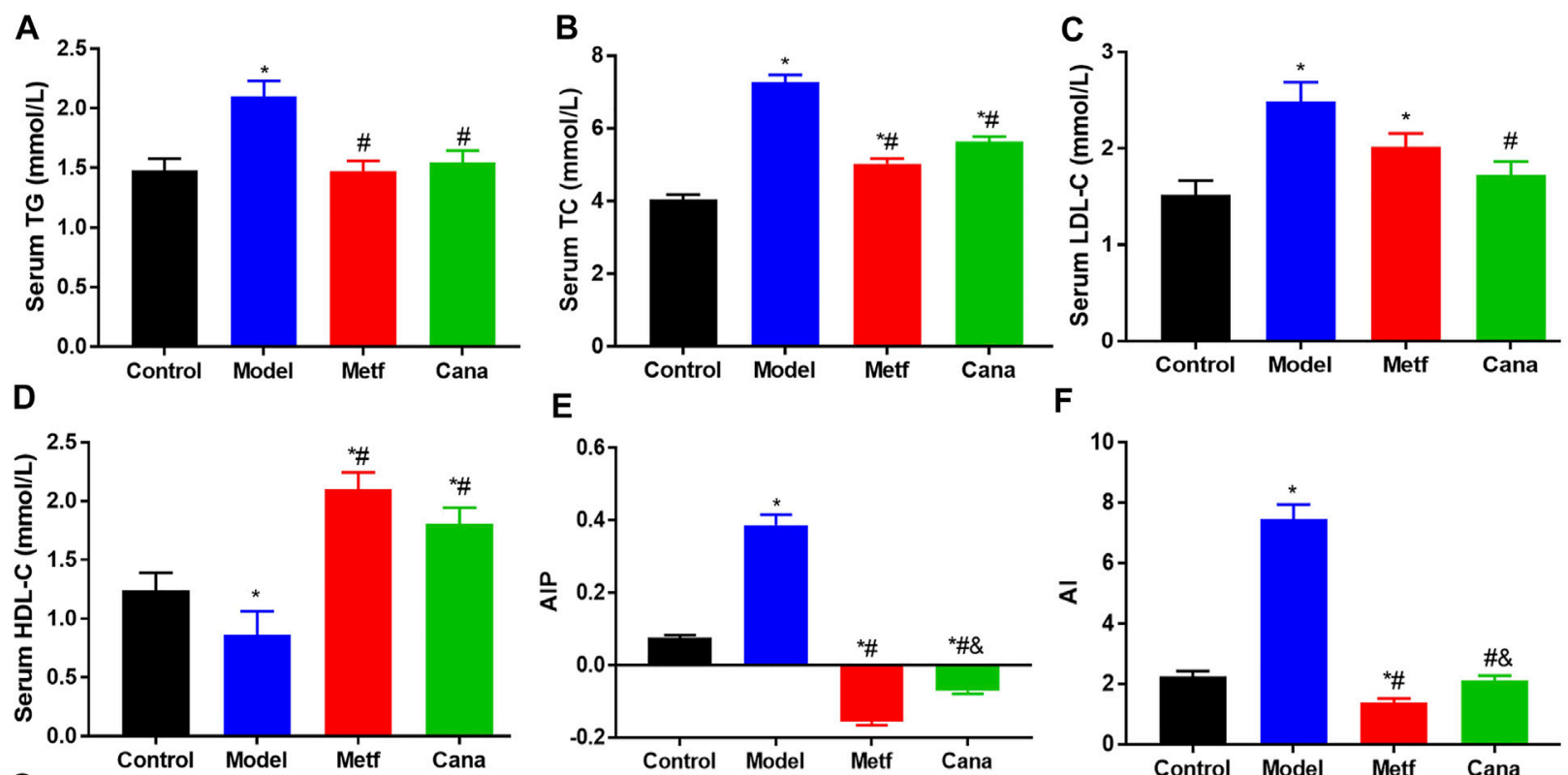

$\mathbf{F}$

G

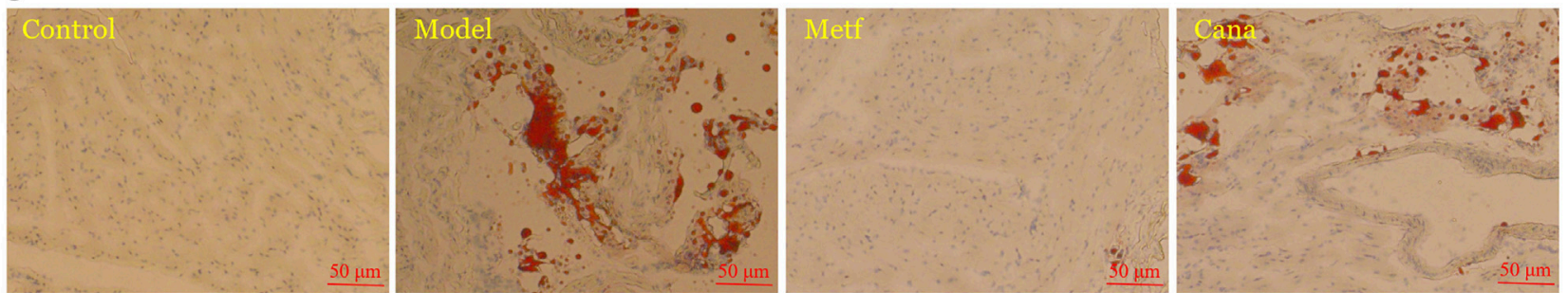

FIGURE 1 | Effects of canagliflozin (Cana) on lipid profile in type 2 diabetes mellitus (T2DM) mice. Triglyceride (TG) (A), total cholesterol (TC) (B), low-density lipoprotein cholesterol (LDL-C) (C), and high-density lipoprotein cholesterol (HDL-C) (D) levels in serum. Atherogenic index of plasma (AIP) (E), calculated by lg(TG / HDL-C). Atherogenic index (Al) (F), calculated by (TC - HDL-C) / HDL-C. Cardiac tissues with Oil Red O staining (G). Data are expressed as mean \pm SEM. ${ }^{\circledR} p<0.05$, the Cana group compared with the Metf group; ${ }^{*} p<0.05$, compared with the model group; ${ }^{*} p<0.05$, compared with the control group. Six mice in each group were randomly selected, and statistical analyses were performed.

\section{Statistical analysis}

Data are presented as the mean \pm standard error of the mean (SEM). The differences between groups were analyzed by GraphPad Prism software using Student's $t$-test, one-way, or two-way ANOVA as appropriate (San Diego, CA, USA). Differences were statistically significant when $p<0.05$. In addition, microbial community $\beta$ - and $\alpha$-diversity were calculated using Chao1, Shannon, and Simpson indices, and Bray-Curtis distances were visualized by PCoA. The LEfSe characterized a value of $p<0.05$ and an LDA score $>2$.

\section{RESULTS}

\section{Effects of Cana on hyperlipemia}

First, we demonstrated that Cana significantly improved blood glucose and insulin homeostasis in mice with T2DM (Supplementary Figure S2). Moreover, Metf treatment remarkedly decreased body weight, body weight gain, and BMI after fed with HFD (Supplementary Figure S3) $(p<$ $0.05)$, while Cana treatment could not. In addition, evidence demonstrates that increased concentrations of cholesterol and TG are causal risk factors for CVD (Nordestgaard and Varbo, 2014). Hyperlipidemia is a vital factor for T2DM-related CVD (Carr and Brunzell, 2004). Thus, managing hyperlipidemia is an effective way to prevent CVD. Our results showed that HFD feeding markedly elevated the levels of TC, TG, and LDL-C in serum $(p<0.05)$ (Figures $\mathbf{1 A - C})$ compared with the control group. However, serous TG, TC, and LDL-C levels in the Cana group were markedly reduced $(p<0.05)$ (Figures $1 \mathrm{~A}-\mathrm{C})$. In addition, serum HDL-C level increased in the Cana and Metf groups compared with the Model group (Figure 1D) $(p<0.05)$. Atherogenic index of plasma (AIP) and atherogenic index (AI) values increased with the elevated CVD risk, which are also highly sensitive markers of lipoprotein profiles in CVD patients (Dobiásová, 2006). Cana decreased the AIP and AI levels in T2DM mice (Figures 1E, F), which demonstrated that Cana relieved CVD-induced hyperlipidemia in mice with T2DM. In addition, Oil Red $\mathrm{O}$ staining indicated that abnormal lipid accumulation was observed in the model group, while treatment with Cana alleviated the lipid accumulation in cardiac tissues (Figure 1G). In addition, we 


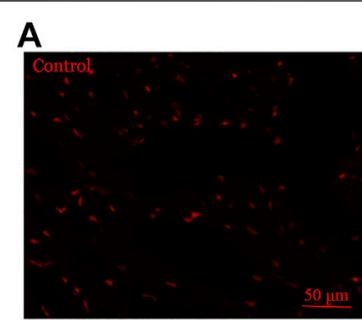

B

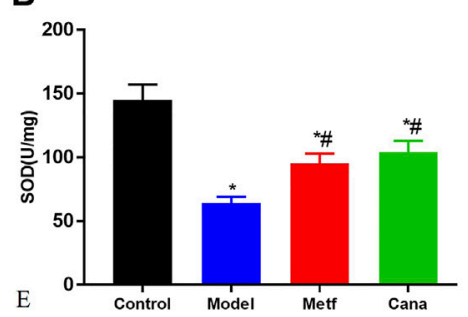

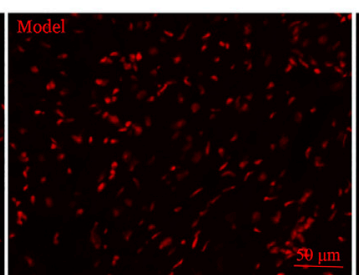

C

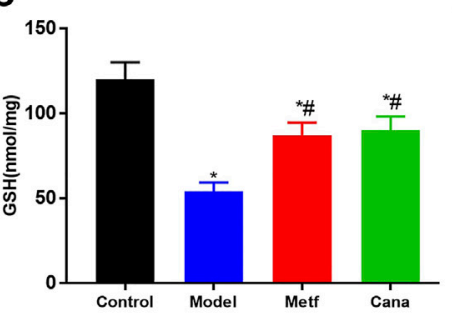

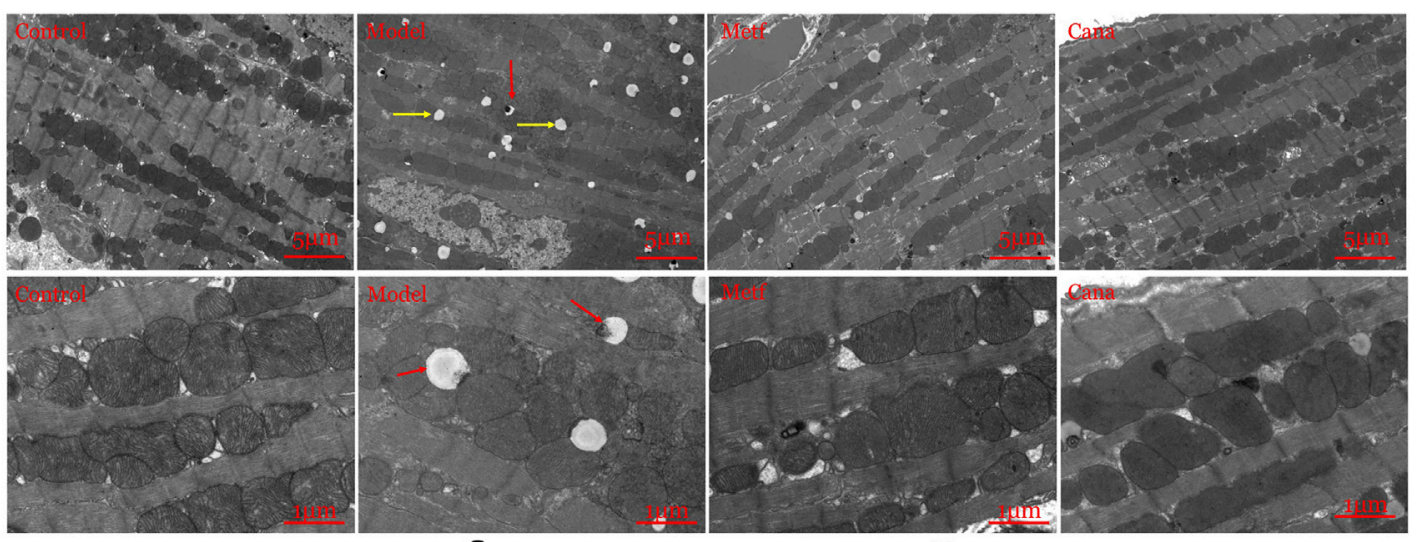

F

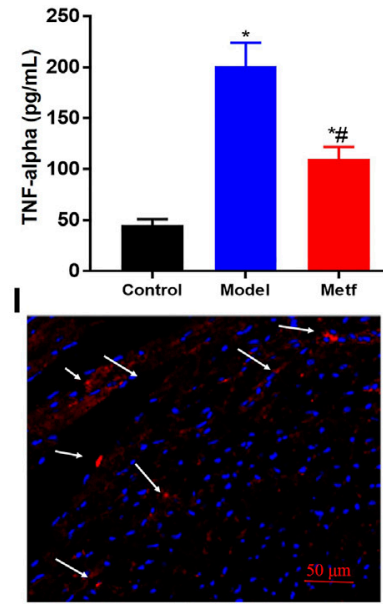

G

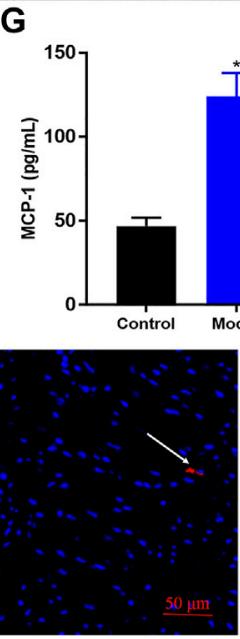

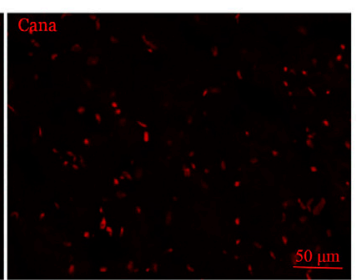

D
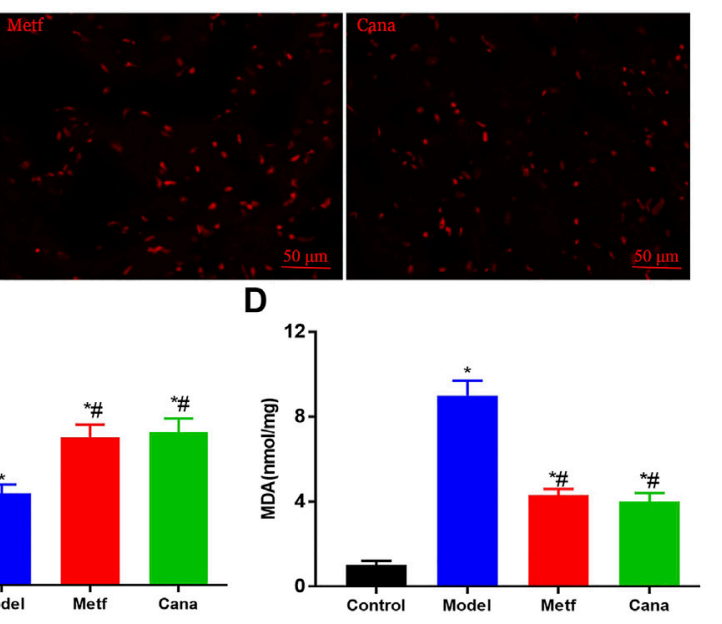

H

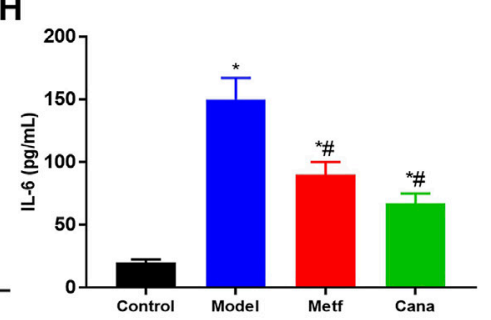

FIGURE 2 | Effects of Cana on alleviating oxidative stress and relieving inflammation. Reactive oxygen species (ROS) in cardiac tissues (A). Superoxide dismutase (SOD) (B), gluthathione (GSH) (C), and malondialdehyde (MDA) (D) contents in cardiac tissues. The regulatory effect of Cana on cardiac mitochondria in myocardial tissues (E). The scale bar is 5 and $1 \mu \mathrm{m}$ in the upper panel and bottom panel, respectively. Serum concentrations of tumor necrosis factor (TNF)-alpha (F), monocyte chemotactic protein 1 (MCP-1) (G), and interleukin 6 (IL-6) (H). The immunofluorescence staining of heme oxygenase 1 (HO-1) in cardiac tissues, scale bar: 50 $\mu$ m (I). Data are expressed as mean \pm SEM. \#p < 0.05, compared with the model group; ${ }^{*} p<0.05$, compared with the control group. Six mice in each group were randomly selected for analyses.

demonstrated the activation of AMPK in cardiac tissues after Cana treatment (Supplementary Figure S4), which may contribute to decrease the lipid levels. Taken together, the above results demonstrated that Cana can perform an antiCVD effect via suppressing lipid accumulation in serum and cardiac tissue in T2DM mice. 
TABLE 1 | Effect of the canagliflozin (Cana) treatment on hematological parameters.

\begin{tabular}{|c|c|c|c|c|}
\hline Variable & Control & Model & Metf & Cana \\
\hline White blood cells (WBCs) (×10\%/L) & $4.6 \pm 0.5$ & $14.1 \pm 0.2^{\star}$ & $8.1 \pm 0.9^{\star \#}$ & $10.0 \pm 1.1^{\star \#}$ \\
\hline Monocytes $\left(\times 10^{9} / \mathrm{L}\right)$ & $0.2 \pm 0.03$ & $0.3 \pm 0.02^{*}$ & $0.6 \pm 0.04^{*}$ & $0.4 \pm 0.02^{* \# \&}$ \\
\hline Monocytes (\%) & $5.0 \pm 0.3$ & $3.8 \pm 0.25^{\star}$ & $7.4 \pm 0.5^{\star \#}$ & $2.5 \pm 0.3^{\star \# \&}$ \\
\hline Lymphocytes (×109/L) & $3.2 \pm 0.24$ & $11.9 \pm 0.9^{\star}$ & $4.9 \pm 0.35^{* \#}$ & $11.2 \pm 1.3^{*} \&$ \\
\hline Lymphocytes (\%) & $67.4 \pm 5.6$ & $76.6 \pm 4.3$ & $60.8 \pm 5.7^{\#}$ & $83.7 \pm 7.6^{\star \# \&}$ \\
\hline Granulocytes (×10\%/L) & $1.3 \pm 0.09$ & $2.3 \pm 0.14^{*}$ & $2.6 \pm 0.15^{\star}$ & $3.5 \pm 0.41^{* \# \&}$ \\
\hline Granulocytes (\%) & $27.5 \pm 2.7$ & $19.5 \pm 1.5^{*}$ & $31.8 \pm 2.9^{\#}$ & $13.7 \pm 1.1^{* \# \&}$ \\
\hline Platelets $\left(\times 10^{9} / \mathrm{L}\right)$ & $1940.5 \pm 124.2$ & $3,307 \pm 32.3^{\star}$ & $2,467 \pm 250.4^{* \#}$ & $2,707 \pm 265 \cdot 1^{*}$ \\
\hline PDW & $17.4 \pm 1.2$ & $17.4 \pm 1.3$ & $18.0 \pm 1.5$ & $19.3 \pm 1.2$ \\
\hline MCV (fl) & $49.5 \pm 5.0$ & $48.5 \pm 5.0$ & $48.7 \pm 3.6$ & $52.7 \pm 4.8$ \\
\hline RDW (\%) & $37.8 \pm 3.2$ & $38.5 \pm 2.8$ & $38.4 \pm 3.4$ & $37.0 \pm 2.8$ \\
\hline Mean platelet volume (MPV) (fl) & $4.75 \pm 0.5$ & $7.56 \pm 0.8^{\star}$ & $6.0 \pm 0.7^{\star \#}$ & $6.3 \pm 0.7^{\star \#}$ \\
\hline
\end{tabular}

Note. PDW, platelet distribution width; RDW, red blood cell distribution width; MCV, mean corpuscular volume. Data are expressed as mean \pm SEM. ${ }^{\&} \mathrm{p}<0.05$, the Cana group compared with the Metf group; $\# \mathrm{p}<0.05$, compared with the model group; ${ }^{*} \mathrm{p}<0.05$, compared with the control group. Six mice in each group were randomly selected, and statistical analyses were performed.

\section{Effects of Cana on oxidative stress and systematic inflammation in type 2 diabetes mellitus mice}

Oxidative stress was increased in T2DM, and this appeared to underlie the development of T2DM and diabetic complications such as CVD (Tangvarasittichai, 2015). Oxidative stress is known as a major contributor to endothelial dysfunction (Magenta et al., 2013), which is a key precursor to the development of CVD (Rocha et al., 2010). The area and intensity of fluorescence from DHE oxidation were notably increased in the model group $(p<0.05)$ (Figure 2A), while Cana or Metf treatments markedly reversed this situation $(p<0.05)$. Notable decreases in the activities of SOD and GSH, while increasing MDA content were observed in the hearts of T2DM mice compared with the normal mice (Figures 2B-D). However, the above situation was conspicuously reversed by Cana supplementation, implying that Cana can effectively relieve myocardial oxidative stress (Figures 2B-D). Importantly, TEM revealed marked muscular fiber twisting, cardiomyocyte dissolution, intercalated disc blurred, and $\mathrm{Z}$ line disappearance. Meanwhile, some myofilaments were flocky in appearance and showed no cross striations in the myocardial tissues of diabetic mice. In addition, mitochondrial crista deformation and damage, mitochondrial autophagy (red arrows presented in Figure 2E, vacuoles with black residue), and fat drop (yellow arrows presented in Figure 2E, vacuoles without black spots) were remarkedly presented in the model group, the effects of which were greatly attenuated by Cana or Metf treatment (Figure 2E), which are beneficial for the suppression of mitochondrial ROS production and subsequently oxidative stress (Zhou et al., 2018).

Chronic inflammation is a mediator of vascular dysfunction in T2DM patients (de Jager et al., 2006; Du et al., 2015; Zhang et al., 2017). Through inflammatory processes, the initial lesion of atherosclerosis and CVD is formed (Libby, 2006). We found that HFD feeding elevated inflammation state by increasing serum levels of TNF-alpha, MCP-1, and IL-6 compared with the control group, whereas Cana administration markedly suppressed this increase in proinflammatory cytokines (Figures 2F-H). Besides, HO-1 level was remarkedly decreased in the model group, while Cana or Metf treatment effectively elevated the HO-1 level in T2DM mice (Figure 2I). Upregulation of HO-1 expression plays an important role in the protective response against oxidative injury and inflammatory effects (Lee and Chau, 2002), which proposes that HO-1 is a promising target protein for therapeutic intervention in CVD. In general, the above results indicate that Cana can effectively alleviate oxidative stress and systematic inflammation by protecting myocardial structural and mitochondrial homeostasis.

\section{Effect of Cana on hematological parameters in type 2 diabetes mellitus mice} Studies demonstrate the strong association between hematological parameters and risk of CVD during systemic inflammation, and hypoxemia is implicated in the pathophysiology mechanisms of CVD (Fan et al., 2018). Our results (Table 1) showed that the white blood cell (WBC) count, platelets, and mean platelet volume (MPV) levels were conspicuously increased in T2DM mice compared with the normal mice, while Cana or Metf treatment effectively reversed this adverse situation. There is a significant association of increased MPV and platelet counts with diabetes related to endothelial dysfunction, coronary artery disease, and its vascular complications (Jabeen et al., 2013; Lippi et al., 2015). Moreover, elevated WBC is a classical inflammatory marker and associated with several CVD risk factors (Yayla et al., 2017). The above results indicate that Cana can conduct an anti-CVD effect via regulating the disorderly hematological parameters.

\section{Effects of Cana on cardiovascular abnormalities in type 2 diabetes mellitus mice}

Normal vasculature is crucial to cardiac function and CVD, which is susceptible to hyperglycemia (Pinter et al., 1999). CD31 plays an important role in endothelial protection, which alleviates the apoptosis of vascular endothelial cells (Evans et al., 2001). HFD- 


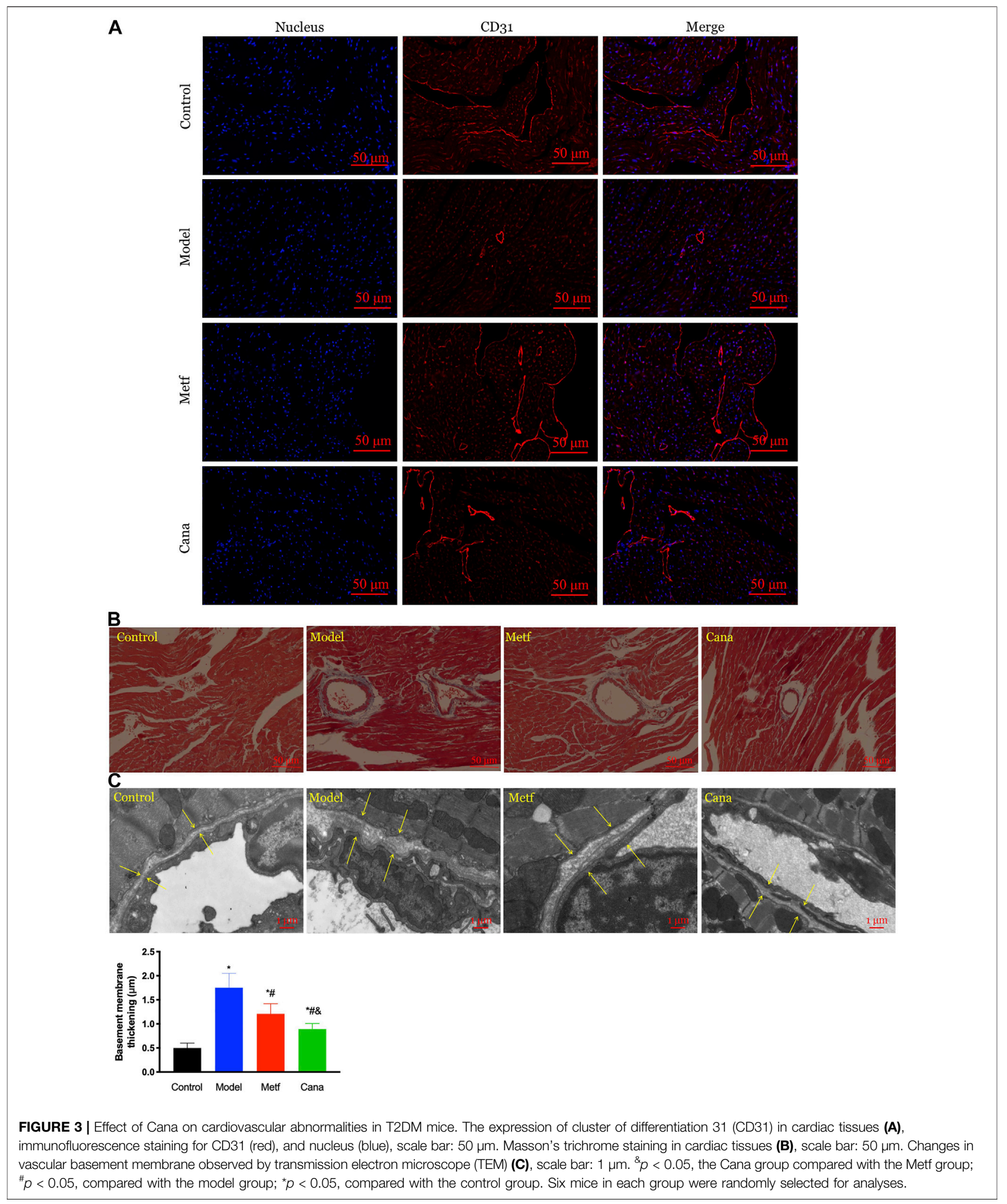




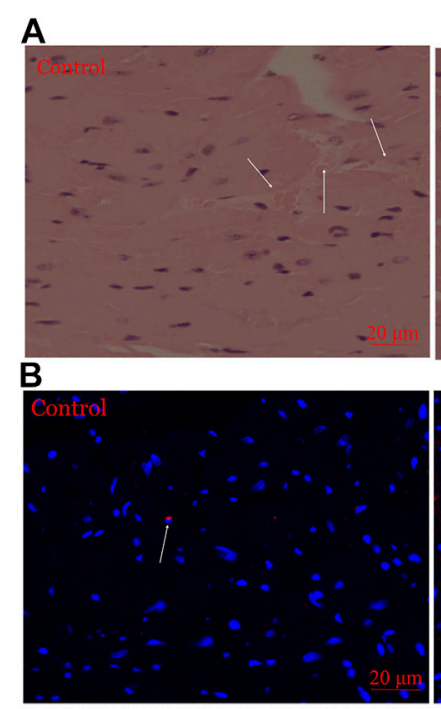

C

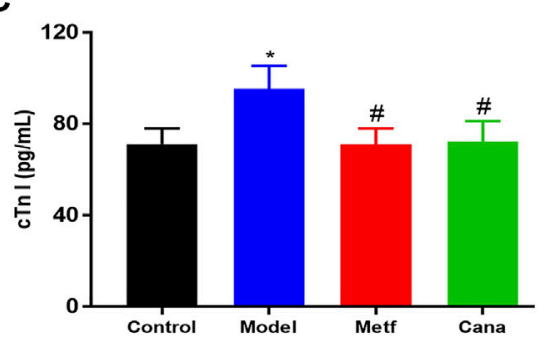

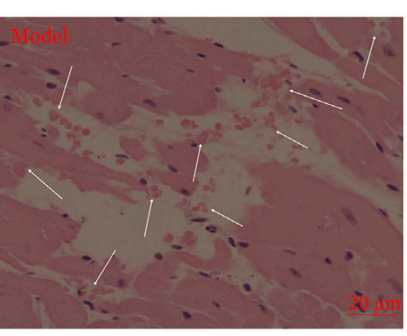
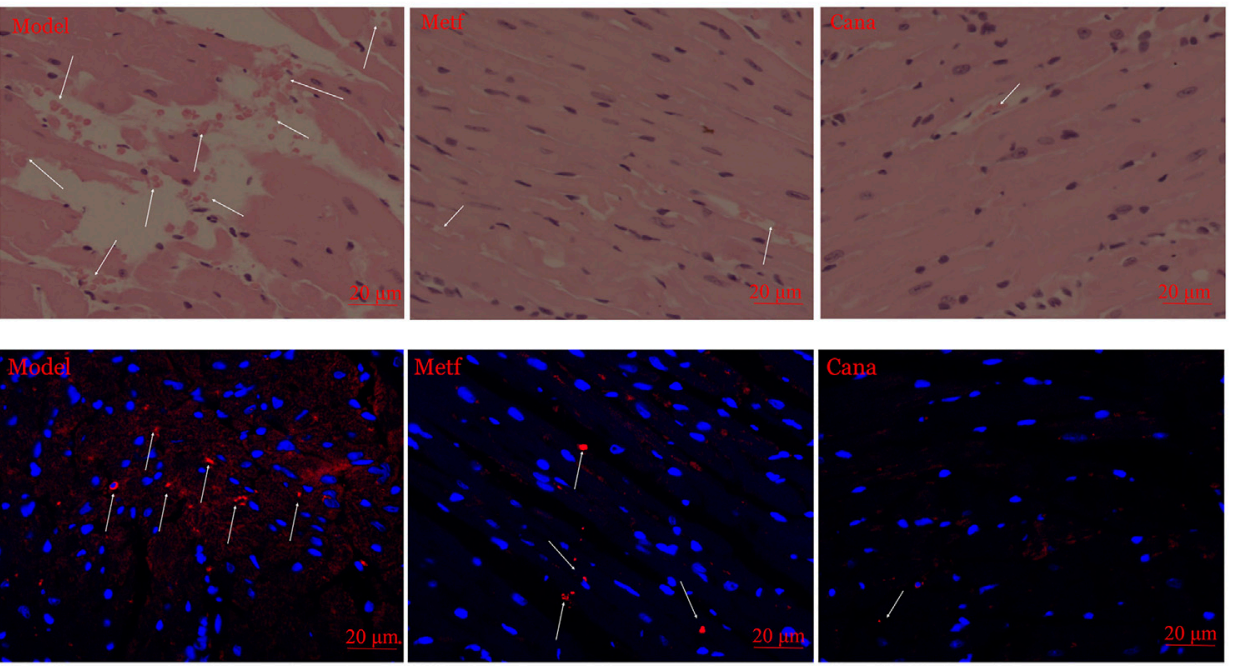

D

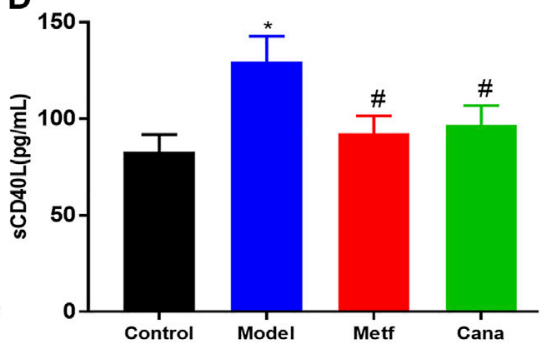

FIGURE 4 | Effects of Cana on improving myocardial injury in T2DM mice. H\&E staining in cardiac tissues (A). TdT-mediated fluorescein nucleotide (cyanine 3dUTP) nick-end labeling (TUNEL) assay to detect apoptosis in cardiac tissues (B), blue represents nucleus, while red represents fragmented DNA, scale bar: 20 mm. Levels of cardiac troponin 1 (cTn I) (C) and soluble cluster of differentiation 40 ligand (sCD4OL) (D) in serum. ${ }^{*} p<0.05$, the Cana group compared with the Metf group; ${ }^{\#} p<0.05$, compared with the model group; ${ }^{*} p<0.05$, compared with the control group. Six mice in each group were randomly selected for analyses.

induced T2DM triggered an overt drop in the number of CD31positive microvessels compared with the normal mice (Figure 3A), while the unfavorable state was reversed by Cana or Metf treatment so as to play a good role in protecting the vascular endothelium. In addition, T2DM-induced basement membrane thickening and microvascular morphology including fibrosis (Figures 3B, C) were largely ameliorated by Cana administration. These data demonstrated a cardioprotective property of Cana by maintaining cardiovascular structural homeostasis.

\section{Effects of Cana on myocardial injury}

Damage to microvessel integrity and cardiac microvascular endothelial cell are considered to be the initial step in vascular complications (Thygesen et al., 2018). Diabetes-induced effects (such as turbulent blood flow and capillary blockage) raised the accumulation of erythrocytes in the microvessel (Zhou et al., 2018), while Cana considerably retarded this negative situation (white arrows presented in Figure 4A). Additionally, Metf or Cana treatment inhibited the diabetes-induced TUNEL-positive cells from increasing (white arrows presented in Figure 4B), supporting the prosurvival capacity of Cana on hyperglycemiamediated cardiac microvascular endothelial cell apoptosis. The above results indicated the beneficial effects of Cana on microvessel integrity, favoring a marked reduction in the risk of cardiac microvascular endothelial cell dysfunction or death in diabetic hearts.

Cardiac troponin is used to diagnose myocardial infarction, and the increased concentration of cardiac troponin indicates the elevated risk for adverse outcomes in individuals (Eggers and Lindahl, 2017). In addition, cTn I, an important subunit of cardiac troponin, is recognized as a marker of myocardial damage (Eggers and Lindahl, 2017). Studies also demonstrated that high levels of sCD40L indicate enhanced inflammatory responses, heightened risk of death, and myocardial infarction in patients (Schönbeck et al., 2001; Heeschen et al., 2003; Cipollone et al., 2003). In our study, levels of serous cTn I and sCD40L in T2DM mice were markedly elevated compared with the control group (Figures 4C, D), while Cana treatment markedly improved the situation. The above results demonstrate the salient effects of Cana on alleviating myocardial injury in T2DM mice.

\section{Cana modulated gut microbiota at various levels}

As shown in Supplementary Table S1, a-diversity (e.g., Good's coverage, Chao1, Shannon, and Simpson indices) of the colonic flora 

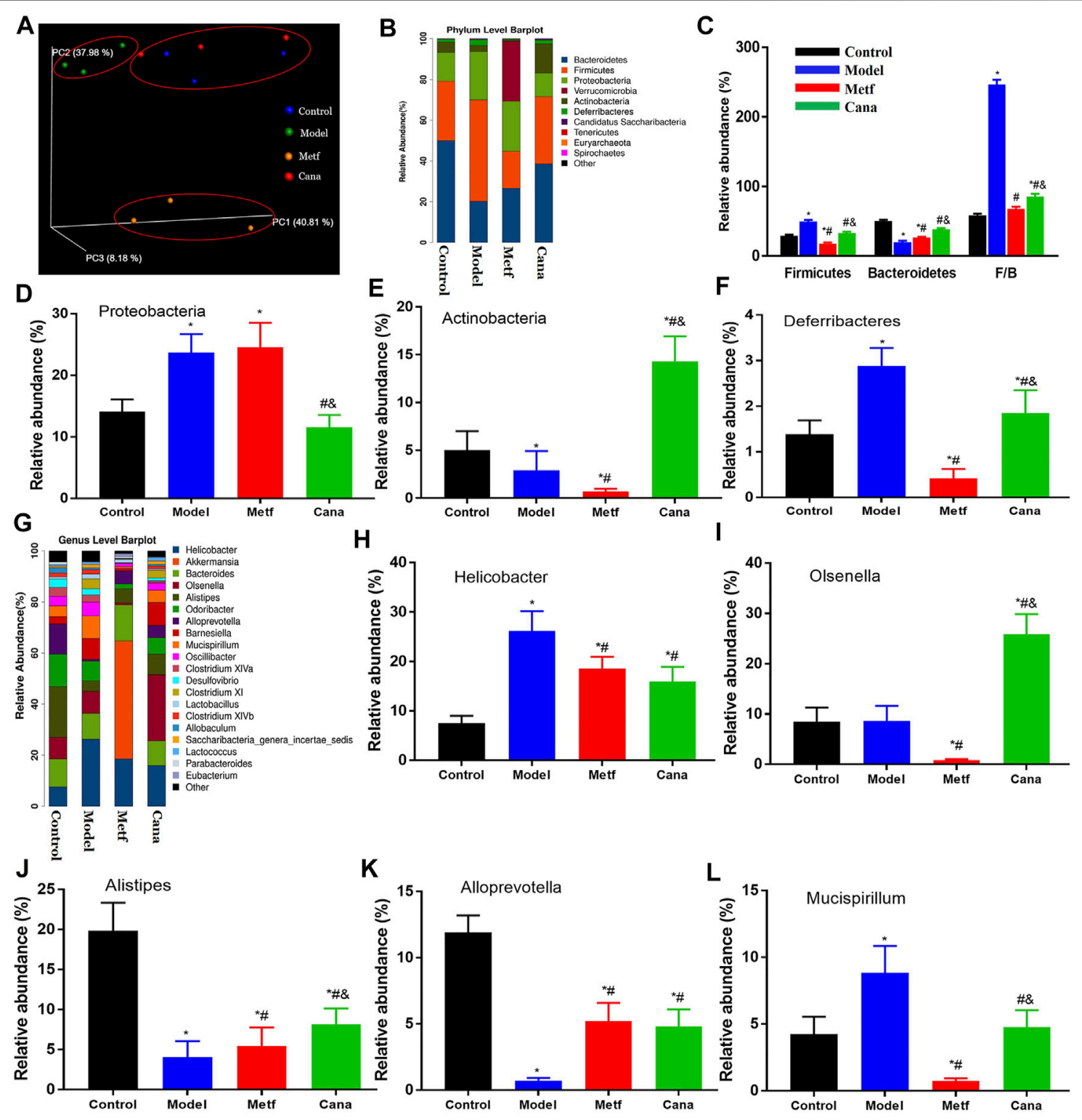

FIGURE 5 | Modulation of microbiota by Cana treatment. PCoA plot of microbiota (A). Abundance at the phylum level (B), including Bacteroidetes and Firmicutes (C), Proteobacteria (D), Actinobacteria (E), and Deferribacteres (F) in various groups. Abundance at the genus level (G), including Helicobacter (H), Olsenella (I), Alistipes (J), Alloprevotella (K), and Mucispirillum (L). Data are expressed as mean \pm SEM. ${ }^{\&} p<0.05$, the Cana group compared with the Metf group; ${ }^{\#} p<0.05$, compared with the model group; ${ }^{\star} p<0.05$, compared with the control group. Three mice in each group were randomly selected for analyses.

in the Cana-treated group was not markedly different compared with the control and model groups. In addition, evaluation of $\beta$ diversity based on nMDS ordination plot (Supplementary Figure S5A), weighted UniFrac heatmap (Supplementary Figure S5B), and weighted PCoA analysis (Figure $\mathbf{5 A}$ ) showed that there were significant differences in colonic flora between the Cana treatment group and model group, and microbial community of the Cana group was similar to the control group. At the phylum level (Figure 5B), the Cana group had a higher abundance of Bacteroidetes with a lower abundance of Firmicutes, and an increased ratio of Firmicutes/Bacteroidetes (Figure 5C) compared with that of the model group. A significant elevation in the relative abundance of Proteobacteria was induced in the model group compared with control group and was remarkedly decreased in the Cana group, however, not reversed by Metf treatment (Figure 5D). In addition, administration of Cana remarkably enriched the abundance of Actinobacteria and decreased the abundance of Deferribacteres in mice with T2DM (Figures 5E, F). The abundance analysis at the genus level indicated that there were conspicuous differences in flora types and abundance in T2DM mice after Cana treatment (Figure 5G). Furthermore, Cana regulated the disorder of microbial community in the genus level as follows: 1. increasing the relative abundance of Olsenella, Alistipes, and Alloprevotella (Figures 5I-K), which may be associated with a 


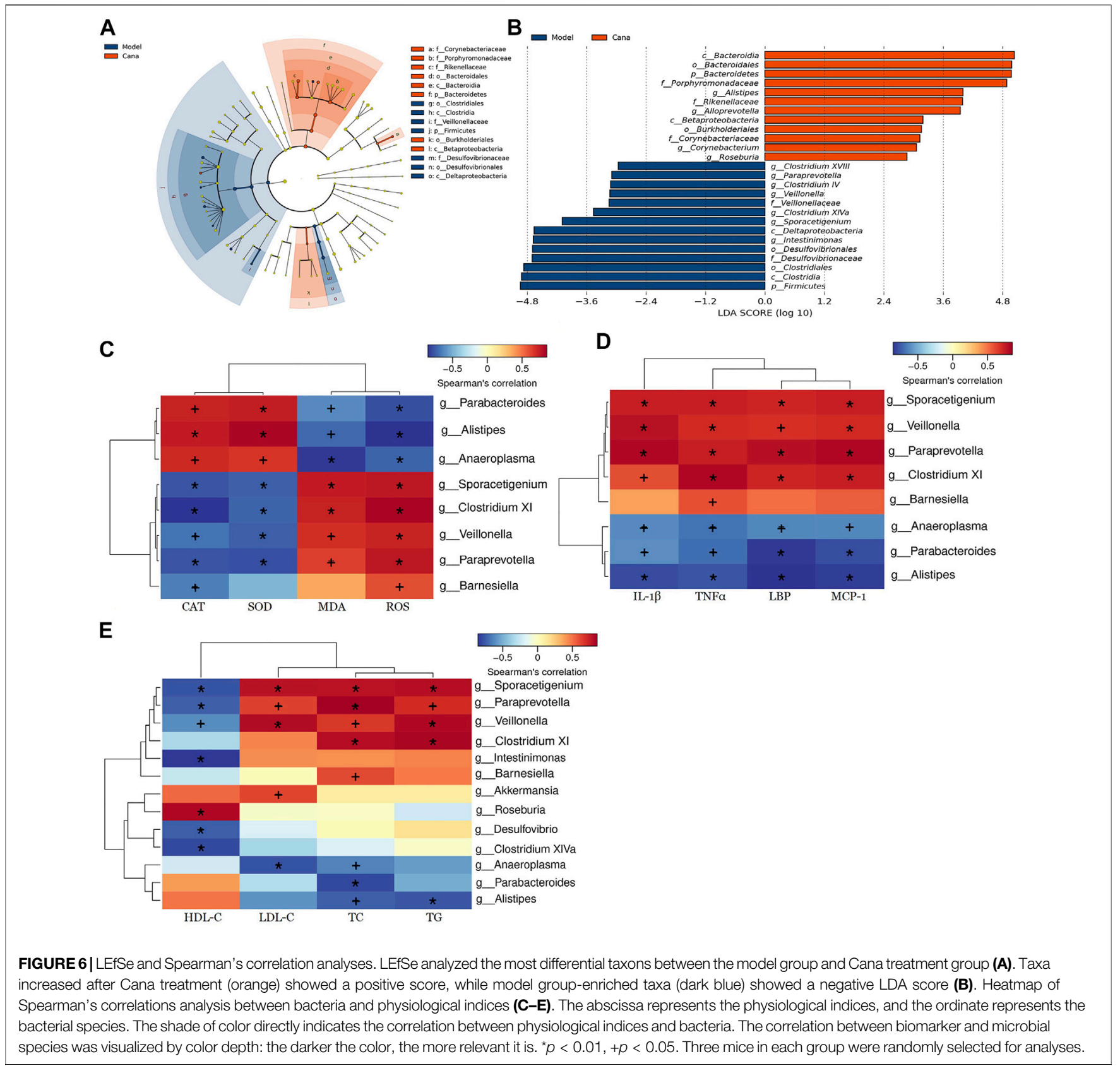

healthier microbiome, as suggested previously (Kong et al., 2019); 2. decreasing the relative abundance of Helicobacter and Mucispirillum (Figures 5H, L), which was positively correlated with TNF-alpha and LPS contents (Li et al., 2019). Altogether, the above results demonstrated that intestinal ecosystem in mice with diabetic CVD were adjusted to a relatively normal state by oral administration of Cana.

\section{Cana induced phylotype changes in gut microbiota}

To further elucidate the effects of Cana treatment on the regulation of various bacterial taxa in the colon, a pairwise comparison between the model and Cana groups was conducted by LEfSe analysis. It indicated that Cana reduced the level of Firmicutes, including Sporacetigenium, Veillonella, and Clostridium XI, while remarkedly elevating Bacteroidetes, including short-chain fatty acid (SCFA) producers such as Alloprevotella and Bacteroides $(p<0.05)$ (Figures 6A,B). The Cana treatment increased the amount of Porphyromonadaceae, Rikenellaceae, and Corynebacteriaceae, while a decrease in Desulfovibrionaceae and Veillonellaceae. At the genus level, the Cana group was marked by a dramatic increase in Alistipes, Roseburia, and Corynebacterium (Figure 6B), which can increase the production of shortchain fatty acids (SCFAs) (Franzosa et al., 2018; Amabebe 


\section{Gut microbiota regulation}

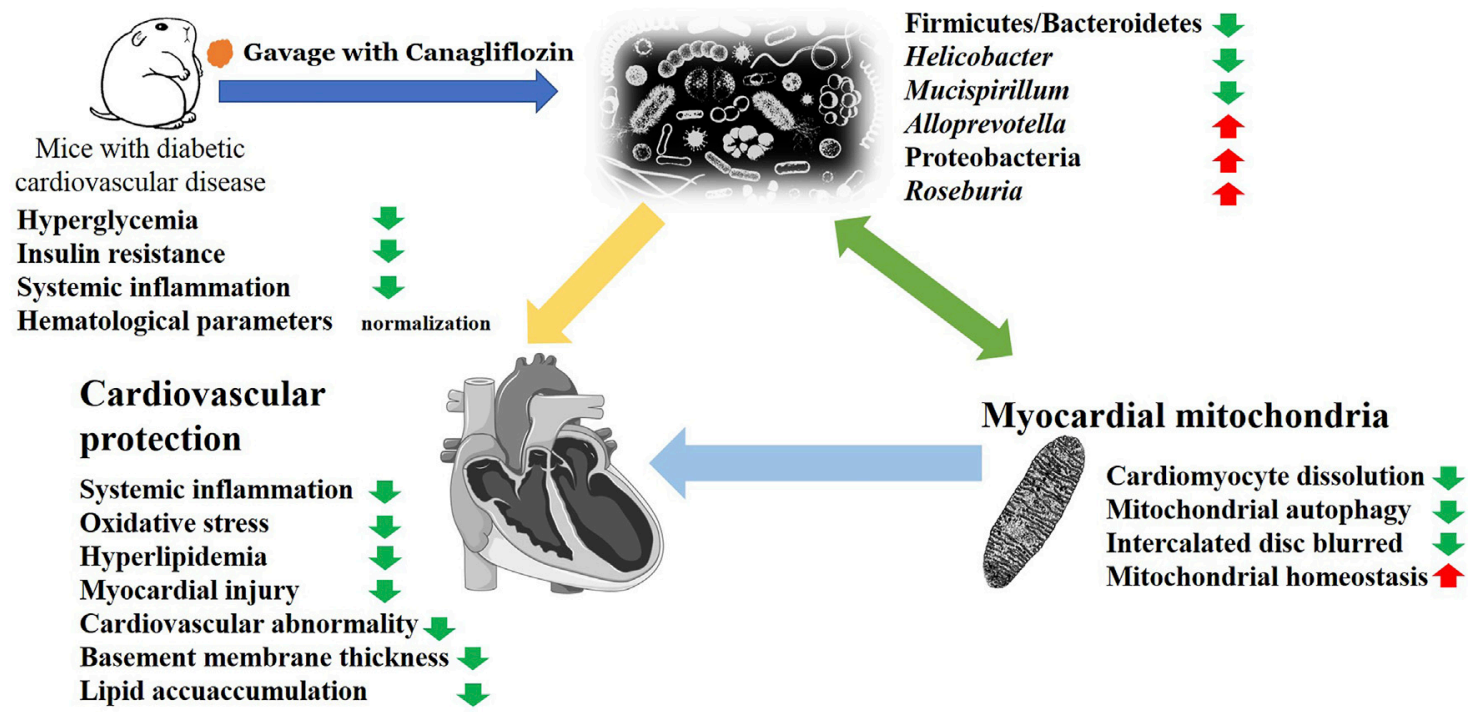

FIGURE 7 | The possible mechanism of Cana on alleviating diabetic cardiovascular disease (CVD). In summary, Cana can effectively alleviate the risk factors for diabetic CVD such as oxidative stress, inflammation, hyperlipemia, atherosclerosis, mitochondrial disorder, thickness of vascular basement membrane, and myocardial injury. The amelioration of gut microbiota and myocardial mitochondrial homeostasis represent an important mechanism underlying the cardiovascular benefits.

and Anumba, 2020). Meanwhile, Paraprevotella, Veillonella, Sporacetigenium, and Intestinimonas were significantly reduced after Cana treatment compared with the model group (Figure 6B). In general, the taxonomical distribution within groups at phylum, family, and genus levels for the colonic samples revealed the divergent composition of communities after Cana treatment in mice with diabetic CVD.

Using Spearman's correlation analysis, relationships of physiological index and bacterial abundance were clarified (Figures 6C-E). At the genus level, Parabacteroides and Alistipes were positively correlated with SOD and CAT, while negatively correlated with IL- $1 \beta$, MDA, ROS, TNFalpha, MCP-1, and LBP levels, and their abundances were increased after Cana treatment. In addition, Clostridium XI, Veillonella, and Paraprevotella were negatively correlated with CAT and SOD, while positively related to MDA, ROS, and the above inflammatory cytokines levels. Meanwhile, Alistipes and Roseburia were negatively correlated with LDL-C, TC, and TG contents, while positively related to HDL-C level. Cana treatment caused an increase in the relative abundances of Alistipes and Roseburia in mice with diabetic CVD. Moreover, Paraprevotella, Veillonella, Clostridium XI, and Barnesiella presented a positive correlation with LDL-C, TC, and TG contents, while negatively related to HDL-C level. These findings indicate that alterations in the gut microbiota after Cana treatment lead to the reduction of systemic inflammation, oxidative stress, and lipid accumulation in mice with diabetic CVD. Collectively, these data reinforce the link between gut dysbiosis and diabetic CVD, and indicate that Cana can manipulate gut microbiota and attenuate the CVD complications of T2DM.

\section{DISCUSSION}

A clinical research indicated that Cana can lower the risk of cardiovascular events in T2DM patients (Perkovic et al., 2019). Meanwhile, the underlying mechanisms were still unclear. Our present study showed that Cana can markedly relieve the oxidative stress and inflammation state, which may be due to the Cana-maintained mitochondrial homeostasis of cardiac tissue in mice with diabetic CVD. Mitochondrial dysfunction resulted in the overproduction of ROS, and cardiovascular function can be improved by relieving mitochondrial dysfunction and its related oxidative stress and systemic inflammatory state (Ren et al., 2018). Further understanding of oxidative stress as well as its downstream effects on cellular function will be conducive to identify more specific targets for CVD treatment (Kayama et al., 2015).

Interestingly, Cana treatment decreased the relative abundance of Firmicutes, while remarkedly raising Bacteroidetes, such as Alloprevotella and Bacteroides, which were beneficial in alleviating hypertension and atherosclerosis (Tang et al., 2017). In addition, Cana administration was marked by a dramatic increase in Roseburia, Alloprevotella, and Bacteroides, which can elevate the production of SCFAs. More importantly, it has been recently confirmed that Alistipes was causally linked to decreased triglyceride concentration in serum (Liu et al., 2022), which was consistent with our present study (Figures 5J, 6E). Thus, gut microbiota may play a vital role in regulating serum lipid composition. Furthermore, Spearman's correlation analysis demonstrated that the colonic microbiota composition altered by Cana treatment was related with the blood lipid, oxidative stress, and inflammation. It has been verified that 
gut microbiota and its metabolites affect the autophagy and damage of the mitochondria, thus, regulating the mitochondrial function of the host (Franco-Obregón and Gilbert, 2017). Therefore, Cana altered colonic microbiota composition compared with the model group in mice with T2DM-related CVD, which contributed to the improvement of mitochondrial homeostasis and the progress of CVD (Jin et al., 2021). It is worth noting that our present results indicated that the effects of Cana on alleviating diabetic CVD were mostly coincidental with that of the Metf group besides the effect on body weight lowering. It showed that Metf treatment significantly reduced the body weight compared with that of the Cana group (Supplementary Figure S3), which may relate with the various colonic microbiota regulation. Importantly, Metf markedly increased the abundance of Akkermansia compared with the Cana group (Figure 5G), which has been verified to decrease body weight in clinical trials (Shin et al., 2014; Depommier et al., 2019). Moreover, the bioavailabilities of Metf, Cana, Empa, and Dapa in humans were about $95 \%, 65 \%, 75 \%$, and $78 \%$, respectively (Beckmann, 1969; Garcia-Ropero et al., 2018). In addition, $81.2 \%$ and $15.3 \%$ of the oral intake of Empa were excreted with feces and urine in male mice as shown in the documents submitted to the FDA for approval (Application Number: 204629Orig1s000). Furthermore, $85.4 \%$ of Cana and its metabolites in male mice were excreted with the feces (Mamidi et al., 2014). While, Metf was mainly excreted through the urine and a small part through the feces (Beckmann, 1969). In general, the different $\mathrm{ADME}$ of the above drugs may result in various bioactivities.

In summary, the primary effects of Cana are its regulation on gut microbiota and myocardial mitochondrial homeostasis, which may play a considerable role in antiCVD (Figure 7). Altogether, our study presents a novel therapeutic role for Cana in T2DM-related CVD. Limitations to this study should be noted. First, further research to clarify the molecular mechanism of the associated changes in colonic microbiota that leads to the improvement of diabetic CVD is still necessary. Second, the relationship between microbiota and CVD should be further clarified utilizing fecal bacteria transplantation in antibiotic-treated or germ-free mice model, which is necessary to be comprehensively addressed in future research. In addition, the most important probiotic strains in alleviating diabetic CVD after Cana treatment were not clarified.

\section{REFERENCES}

Amabebe, E., and Anumba, D. O. C. (2020). Female Gut and Genital Tract Microbiota-Induced Crosstalk and Differential Effects of Short-Chain Fatty Acids on Immune Sequelae. Front. Immunol. 11, 2184. doi:10.3389/fimmu. 2020.02184

Arjinajarn, P., Pongchaidecha, A., Chueakula, N., Jaikumkao, K., Chatsudthipong, V., Mahatheeranont, S., et al. (2016). Riceberry Bran Extract Prevents Renal Dysfunction and Impaired Renal Organic Anion Transporter 3 (Oat3) Function by Modulating the PKC/Nrf2 Pathway in Gentamicin-Induced Nephrotoxicity in Rats. Phytomedicine 23 (14), 1753-1763. doi:10.1016/j. phymed.2016.10.014

\section{DATA AVAILABILITY STATEMENT}

The original contributions presented in the study are publicly available. The data can be found here: https://www.ncbi.nlm.nih. gov/sra/PRJNA795843.

\section{ETHICS STATEMENT}

The animal study was reviewed and approved by the Experimental Animal Care and Use Committee of the Ocean University of China.

\section{AUTHOR CONTRIBUTIONS}

XW conducted the research and wrote the paper. DL and ZW helped with the animal experiments and biochemical analysis. XW, HJ, and GY analyzed the experimental data. XW, CC, HJ, GL, and GY contributed to the manuscript revision. GY, HJ, and XW designed and directed the project. All authors read and approved the final manuscript.

\section{FUNDING}

This work was supported by the National Natural Science Foundation of China (81991522, 81402982, 21807094), National Science and Technology Major Project of China (2018ZX09735-004), Shandong Provincial Major Science and Technology Innovation Project (2018SDKJ0404), Taishan Scholar Climbing Project (TSPD20210304), Project funded by the China Postdoctoral Science Foundation (2021M703684), Fundamental Research Funds for the Central Universities (202042005), and Natural Science Foundation of Shandong Province (ZR2017BC007).

\section{SUPPLEMENTARY MATERIAL}

The Supplementary Material for this article can be found online at: https://www.frontiersin.org/articles/10.3389/fphar.2022.839640/ full\#supplementary-material

Aronson, D., and Rayfield, E. J. (2002). How Hyperglycemia Promotes Atherosclerosis: Molecular Mechanisms. Cardiovasc. Diabetol. 1 (1), 1. doi:10.1186/1475-2840-1-1

Battson, M. L., Lee, D. M., Jarrell, D. K., Hou, S., Ecton, K. E., Weir, T. L., et al. (2017). Suppression of Gut Dysbiosis Reverses Western Diet-Induced Vascular Dysfunction. Am. J. Physiol. Endocrinol. Metab. 314 (5), E468-E477. doi:10. 1152/ajpendo.00187.2017

Beckmann, R. d. (1969). Absorption, Distribution in the Organism and Elimination of Metformin. Diabetologia 5 (5), 318-324. doi:10.1007/ bf00452906

Boulangé, C. L., Neves, A. L., Chilloux, J., Nicholson, J. K., and Dumas, M. E. (2016). Impact of the Gut Microbiota on Inflammation, Obesity, and Metabolic Disease. Genome Med. 8 (1), 42-12. doi:10.1186/s13073-016-0303-2 
Braunwald, E. (2008). Biomarkers in Heart Failure. N. Engl. J. Med. 358 (20), 2148-2159. doi:10.1056/nejmra0800239

Brindle, P., Beswick, A., Fahey, T., and Ebrahim, S. (2006). Accuracy and Impact of Risk Assessment in the Primary Prevention of Cardiovascular Disease: a Systematic Review. Heart 92 (12), 1752-1759. doi:10.1136/hrt.2006.087932

Carr, M. C., and Brunzell, J. D. (2004). Abdominal Obesity and Dyslipidemia in the Metabolic Syndrome: Importance of Type 2 Diabetes and Familial Combined Hyperlipidemia in Coronary Artery Disease Risk. J. Clin. Endocrinol. Metab. 89 (6), 2601-2607. doi:10.1210/jc.2004-0432

Cipollone, F., Ferri, C., Desideri, G., Paloscia, L., Materazzo, G., Mascellanti, M., et al. (2003). Preprocedural Level of Soluble CD40L Is Predictive of Enhanced Inflammatory Response and Restenosis after Coronary Angioplasty. Circulation 108 (22), 2776-2782. doi:10.1161/01.CIR.0000103700.05109.0D

Das, N., Mandala, A., Naaz, S., Giri, S., Jain, M., Bandyopadhyay, D., et al. (2017). Melatonin Protects against Lipid-Induced Mitochondrial Dysfunction in Hepatocytes and Inhibits Stellate Cell Activation during Hepatic Fibrosis in Mice. J. Pineal Res. 62 (4), e12404. doi:10.1111/jpi.12404

de Jager, J., Dekker, J. M., Kooy, A., Kostense, P. J., Nijpels, G., Heine, R. J., et al. (2006). Endothelial Dysfunction and Low-Grade Inflammation Explain Much of the Excess Cardiovascular Mortality in Individuals with Type 2 Diabetes: the Hoorn Study. Arterioscler Thromb. Vasc. Biol. 26 (5), 1086-1093. doi:10.1161/ 01.ATV.0000215951.36219.a4

DeFronzo, R. A., Ferrannini, E., Groop, L., Henry, R. R., Herman, W. H., Holst, J. J., et al. (2015). Type 2 Diabetes Mellitus. Nat. Rev. Dis. Primers 1, 15019. doi:10. 1038/nrdp.2015.19

Depommier, C., Everard, A., Druart, C., Plovier, H., Van Hul, M., Vieira-Silva, S., et al. (2019). Supplementation with Akkermansia Muciniphila in Overweight and Obese Human Volunteers: a Proof-Of-Concept Exploratory Study. Nat. Med. 25 (7), 1096-1103. doi:10.1038/s41591-019-0495-2

Dobiásová, M. (2006). AIP-atherogenic index of Plasma as a Significant Predictor of Cardiovascular Risk: From Research to Practice. Vnitrní Lékarství 52 (1), 64-71.

Du, B., Ouyang, A., Eng, J. S., and Fleenor, B. S. (2015). Aortic Perivascular Adipose-Derived Interleukin-6 Contributes to Arterial Stiffness in Low-Density Lipoprotein Receptor Deficient Mice. Am. J. Physiol. Heart Circ. Physiol. 308 (11), H1382-H1390. doi:10.1152/ajpheart.00712.2014

Dziuba, J., Alperin, P., Racketa, J., Iloeje, U., Goswami, D., Hardy, E., et al. (2014). Modeling Effects of SGLT-2 Inhibitor Dapagliflozin Treatment versus Standard Diabetes Therapy on Cardiovascular and Microvascular Outcomes. Diabetes Obes. Metab. 16 (7), 628-635. doi:10.1111/dom.12261

Eggers, K. M., and Lindahl, B. (2017). Application of Cardiac Troponin in Cardiovascular Diseases Other Than Acute Coronary Syndrome. Clin. Chem. 63 (1), 223-235. doi:10.1373/clinchem.2016.261495

El-Azab, M. F., Hazem, R. M., and Moustafa, Y. M. (2012). Role of Simvastatin And/or Antioxidant Vitamins in Therapeutic Angiogenesis in Experimental Diabetic Hindlimb Ischemia: Effects on Capillary Density, Angiogenesis Markers, and Oxidative Stress. Eur. J. Pharmacol. 690 (1), 31-41. doi:10. 1016/j.ejphar.2012.06.002

Evans, P. C., Taylor, E. R., and Kilshaw, P. J. (2001). Signaling through CD31 Protects Endothelial Cells from Apoptosis. Transplantation 71 (3), 457-460. doi:10.1097/00007890-200102150-00020

Fan, L., Gui, L., Chai, E. Q., and Wei, C. J. (2018). Routine Hematological Parameters Are Associated with Short- and Long-Term Prognosis of Patients with Ischemic Stroke. J. Clin. Lab. Anal. 32 (2), e22244. doi:10. 1002/jcla.22244

Fei, N., and Zhao, L. (2013). An Opportunistic Pathogen Isolated from the Gut of an Obese Human Causes Obesity in Germfree Mice. ISME J. 7 (4), 880-884. doi:10.1038/ismej.2012.153

Figueiredo, V. N., Yugar-Toledo, J. C., Martins, L. C., Martins, L. B., de Faria, A. P., de Haro Moraes, C., et al. (2012). Vascular Stiffness and Endothelial Dysfunction: Correlations at Different Levels of Blood Pressure. Blood Press. 21 (1), 31-38. doi:10.3109/08037051.2011.617045

Fischer, A. H., Jacobson, K. A., Rose, J., and Zeller, R. (2008). Hematoxylin and Eosin Staining of Tissue and Cell Sections. CSH Protoc. 2008 (5), pdb.prot4986. doi:10.1101/pdb.prot 4986

Forslund, K., Hildebrand, F., Nielsen, T., Falony, G., Le Chatelier, E., Sunagawa, S., et al. (2015). Disentangling Type 2 Diabetes and Metformin Treatment
Signatures in the Human Gut Microbiota. Nature 528 (7581), 262-266. doi:10.1038/nature15766

Fox, C. S., Golden, S. H., Anderson, C., Bray, G. A., Burke, L. E., De Boer, I. H., et al. (2015). Update on Prevention of Cardiovascular Disease in Adults with Type 2 Diabetes Mellitus in Light of Recent Evidence: a Scientific Statement from the American Heart Association and the American Diabetes Association. Circulation 132 (8), 691-718. doi:10.1161/CIR.0000000000000230

Franco-Obregón, A., and Gilbert, J. A. (2017). The Microbiome-Mitochondrion Connection: Common Ancestries, Common Mechanisms, Common Goals. mSystems 2 (3), e00018-17. doi:10.1128/mSystems.00018-17

Franzosa, E. A., Sirota-Madi, A., Avila-Pacheco, J., Fornelos, N., Haiser, H. J., Reinker, S., et al. (2018). Gut Microbiome Structure and Metabolic Activity in Inflammatory Bowel Disease. Nat. Microbiol. 4 (2), 293-305. doi:10.1038/ s41564-018-0306-4

Fraulob, J. C., Ogg-Diamantino, R., Fernandes-Santos, C., Aguila, M. B., and Mandarim-de-Lacerda, C. A. (2010). A Mouse Model of Metabolic Syndrome: Insulin Resistance, Fatty Liver and Non-alcoholic Fatty Pancreas Disease (NAFPD) in C57BL/6 Mice Fed a High Fat Diet. J. Clin. Biochem. Nutr. 46, 212-223. doi:10.3164/jcbn.09-83

Fung, C. S., Wan, E. Y., Wong, C. K., Jiao, F., and Chan, A. K. (2015). Effect of Metformin Monotherapy on Cardiovascular Diseases and Mortality: a Retrospective Cohort Study on Chinese Type 2 Diabetes Mellitus Patients. Cardiovasc. Diabetol. 14 (1), 137. doi:10.1186/s12933-015-0304-2

Garcia-Ropero, A., Badimon, J. J., and Santos-Gallego, C. G. (2018). The Pharmacokinetics and Pharmacodynamics of SGLT2 Inhibitors for Type 2 Diabetes Mellitus: the Latest Developments. Expert Opin. Drug Metab. Toxicol. 14 (12), 1287-1302. doi:10.1080/17425255.2018.1551877

Garvey, W. T., Van Gaal, L., Leiter, L. A., Vijapurkar, U., List, J., Cuddihy, R., et al. (2018). Effects of Canagliflozin versus Glimepiride on Adipokines and Inflammatory Biomarkers in Type 2 Diabetes. Metabolism 85, 32-37. doi:10. 1016/j.metabol.2018.02.002

Gerstein, H. C., Pogue, J., Mann, J. F., Lonn, E., Dagenais, G. R., McQueen, M., et al. (2005). The Relationship between Dysglycaemia and Cardiovascular and Renal Risk in Diabetic and Non-diabetic Participants in the HOPE Study: a Prospective Epidemiological Analysis. Diabetologia 48 (9), 1749-1755. doi:10.1007/s00125-005-1858-4

Goldner, J. (1938). A Modification of the Masson Trichrome Technique for Routine Laboratory Purposes. Am. J. Pathol. 14 (2), 237-243.

Group, A. C., Patel, A., MacMahon, S., Chalmers, J., Neal, B., Billot, L., et al. (2008). Intensive Blood Glucose Control and Vascular Outcomes in Patients with Type 2 Diabetes. N. Engl. J. Med. 358 (24), 2560-2572. doi:10.1056/NEJMoa0802987

Hawley, S. A., Ford, R. J., Smith, B. K., Gowans, G. J., Mancini, S. J., Pitt, R. D., et al. (2016). The Na+/Glucose Cotransporter Inhibitor Canagliflozin Activates AMPK by Inhibiting Mitochondrial Function and Increasing Cellular AMP Levels. Diabetes 65 (9), 2784-2794. doi:10.2337/db16-0058

Heeschen, C., Dimmeler, S., Hamm, C. W., van den Brand, M. J., Boersma, E., Zeiher, A. M., et al. (2003). Soluble CD40 Ligand in Acute Coronary Syndromes. N. Engl. J. Med. 348 (12), 1104-1111. doi:10.1056/NEJMoa022600

Jabeen, F., Fawwad, A., Rizvi, H. A., and Alvi, F. (2013). Role of Platelet Indices, Glycemic Control and Hs-CRP in Pathogenesis of Vascular Complications in Type-2 Diabetic Patients. Pak J. Med. Sci. 29 (1), 152-156. doi:10.12669/pjms. 291.2592

Jaikumkao, K., Pongchaidecha, A., Chueakula, N., Thongnak, L., Wanchai, K., Chatsudthipong, V., et al. (2018). Renal Outcomes with Sodium Glucose Cotransporter 2 (SGLT2) Inhibitor, Dapagliflozin, in Obese InsulinResistant Model. Biochim. Biophys. Acta Mol. Basis Dis. 1864 (6 Pt A), 2021-2033. doi:10.1016/j.bbadis.2018.03.017

Jain, K. S., Kathiravan, M. K., Somani, R. S., and Shishoo, C. J. (2007). The Biology and Chemistry of Hyperlipidemia. Bioorg. Med. Chem. 15 (14), 4674-4699. doi:10.1016/j.bmc.2007.04.031

Jiang, Y., Zheng, R., and Sang, H. (2021). Cost-Effectiveness of Adding SGLT2 Inhibitors to Standard Treatment for Heart Failure with Reduced Ejection Fraction Patients in China. Front. Pharmacol. 12 (3111), 733681. doi:10.3389/ fphar.2021.733681

Jin, L., Shi, X., Yang, J., Zhao, Y., Xue, L., Xu, L., et al. (2021). Gut Microbes in Cardiovascular Diseases and Their Potential Therapeutic Applications. Protein Cell 12 (5), 346-359. doi:10.1007/s13238-020-00785-9 
Kayama, Y., Raaz, U., Jagger, A., Adam, M., Schellinger, I. N., Sakamoto, M., et al. (2015). Diabetic Cardiovascular Disease Induced by Oxidative Stress. Int. J. Mol. Sci. 16 (10), 25234-25263. doi:10.3390/ijms161025234

Kong, C., Gao, R., Yan, X., Huang, L., and Qin, H. (2019). Probiotics Improve Gut Microbiota Dysbiosis in Obese Mice Fed a High-Fat or High-Sucrose Diet. Nutrition 60, 175-184. doi:10.1016/j.nut.2018.10.002

Lee, D. M., Battson, M. L., Jarrell, D. K., Hou, S., Ecton, K. E., Weir, T. L., et al. (2018). SGLT2 Inhibition via Dapagliflozin Improves Generalized Vascular Dysfunction and Alters the Gut Microbiota in Type 2 Diabetic Mice. Cardiovasc. Diabetol. 17 (1), 62. doi:10.1186/s12933-018-0708-x

Lee, T. S., and Chau, L. Y. (2002). Heme Oxygenase-1 Mediates the Antiinflammatory Effect of Interleukin-10 in Mice. Nat. Med. 8 (3), 240-246. doi:10.1038/nm0302-240

Li, K., Zhang, L., Xue, J., Yang, X., Dong, X., Sha, L., et al. (2019). Dietary Inulin Alleviates Diverse Stages of Type 2 Diabetes Mellitus via Anti-inflammation and Modulating Gut Microbiota in Db/db Mice. Food Funct. 10 (4), 1915-1927. doi:10.1039/c8fo02265h

Liakos, A., Karagiannis, T., Athanasiadou, E., Sarigianni, M., Mainou, M., Papatheodorou, K., et al. (2014). Efficacy and Safety of Empagliflozin for Type 2 Diabetes: a Systematic Review and Meta-Analysis. Diabetes Obes. Metab. 16 (10), 984-993. doi:10.1111/dom.12307

Libby, P. (2006). Inflammation and Cardiovascular Disease Mechanisms. Am. J. Clin. Nutr. 83 (2), 456S-460S. doi:10.1093/ajcn/83.2.456S

Lippi, G., Salvagno, G. L., Nouvenne, A., Meschi, T., Borghi, L., and Targher, G. (2015). The Mean Platelet Volume Is Significantly Associated with Higher Glycated Hemoglobin in a Large Population of Unselected Outpatients. Prim. Care Diabetes 9 (3), 226-230. doi:10.1016/j.pcd.2014.08.002

Liu, X., Tong, X., Zou, Y., Lin, X., Zhao, H., Tian, L., et al. (2022). Mendelian Randomization Analyses Support Causal Relationships between Blood Metabolites and the Gut Microbiome. Nat. Genet. 54, 52-61. doi:10.1038/ s41588-021-00968-y

Magenta, A., Greco, S., Gaetano, C., and Martelli, F. (2013). Oxidative Stress and microRNAs in Vascular Diseases. Int. J. Mol. Sci. 14 (9), 17319-17346. doi:10. 3390/ijms140917319

Mahaffey, K. W., Neal, B., Perkovic, V., de Zeeuw, D., Fulcher, G., Erondu, N., et al. (2018). Canagliflozin for Primary and Secondary Prevention of Cardiovascular Events: Results from the CANVAS Program (Canagliflozin Cardiovascular Assessment Study). Circulation 137 (4), 323-334. doi:10.1161/ CIRCULATIONAHA.117.032038

Mamidi, R. N., Cuyckens, F., Chen, J., Scheers, E., Kalamaridis, D., Lin, R., et al. (2014). Metabolism and Excretion of Canagliflozin in Mice, Rats, Dogs, and Humans. Drug Metab. Dispos 42 (5), 903-916. doi:10.1124/dmd.113.056440

Mishima, E., Fukuda, S., Kanemitsu, Y., Saigusa, D., Mukawa, C., Asaji, K., et al. (2017). Canagliflozin Reduces Plasma Uremic Toxins and Alters the Intestinal Microbiota Composition in a Chronic Kidney Disease Mouse Model. Am. J. Physiol. Ren. Physiol 315 (4), F824-F833. doi:10.1152/ajprenal.00314.2017

Neal, B., Perkovic, V., Mahaffey, K. W., de Zeeuw, D., Fulcher, G., Erondu, N., et al. (2017). Canagliflozin and Cardiovascular and Renal Events in Type 2 Diabetes. N. Engl. J. Med. 377 (7), 644-657. doi:10.1056/NEJMoa1611925

Nishikawa, T., and Araki, E. (2007). Impact of Mitochondrial ROS Production in the Pathogenesis of Diabetes Mellitus and its Complications. Antioxid. Redox Signal. 9 (3), 343-353. doi:10.1089/ars.2006.1458

Nordestgaard, B. G., and Varbo, A. (2014). Triglycerides and Cardiovascular Disease. Lancet 384 (9943), 626-635. doi:10.1016/S0140-6736(14)61177-6

Perkovic, V., Jardine, M. J., Neal, B., Bompoint, S., Heerspink, H. J. L., Charytan, D. M., et al. (2019). Canagliflozin and Renal Outcomes in Type 2 Diabetes and Nephropathy. N. Engl. J. Med. 380 (24), 2295-2306. doi:10.1056/ NEJMoa1811744

Pinter, E., Mahooti, S., Wang, Y., Imhof, B. A., and Madri, J. A. (1999). Hyperglycemia-induced Vasculopathy in the Murine Vitelline Vasculature: Correlation with PECAM-1/CD31 Tyrosine Phosphorylation State. Am. J. Pathol. 154 (5), 1367-1379. doi:10.1016/S0002-9440(10)65391-6

Ren, J., Sowers, J. R., and Zhang, Y. (2018). Metabolic Stress, Autophagy, and Cardiovascular Aging: from Pathophysiology to Therapeutics. Trends Endocrinol. Metab. 29 (10), 699-711. doi:10.1016/j.tem.2018.08.001

Rocha, M., Apostolova, N., Hernandez-Mijares, A., Herance, R., and Victor, V. M. (2010). Oxidative Stress and Endothelial Dysfunction in Cardiovascular
Disease: Mitochondria-Targeted Therapeutics. Curr. Med. Chem. 17 (32), 3827-3841. doi:10.2174/092986710793205444

Rosenwasser, R. F., Sultan, S., Sutton, D., Choksi, R., and Epstein, B. J. (2013). SGLT-2 Inhibitors and Their Potential in the Treatment of Diabetes. Diabetes Metab. Syndr. Obes. 6, 453-467. doi:10.2147/DMSO.S34416

Schönbeck, U., Varo, N., Libby, P., Buring, J., and Ridker, P. M. (2001). Soluble CD40L and Cardiovascular Risk in Women. Circulation 104 (19), 2266-2268. doi:10.1161/hc4401.099447

Shin, N. R., Lee, J. C., Lee, H. Y., Kim, M. S., Whon, T. W., Lee, M. S., et al. (2014). An Increase in the Akkermansia Spp. Population Induced by Metformin Treatment Improves Glucose Homeostasis in Diet-Induced Obese Mice. Gut 63 (5), 727-735. doi:10.1136/gutjnl-2012-303839

Skyler, J. S., Bergenstal, R., Bonow, R. O., Buse, J., Deedwania, P., Gale, E. A., et al. (2009). Intensive Glycemic Control and the Prevention of Cardiovascular Events: Implications of the ACCORD, ADVANCE, and VA Diabetes Trials: a Position Statement of the American Diabetes Association and a Scientific Statement of the American College of Cardiology Foundation and the American Heart Association. Diabetes Care 32 (3), 187-192. doi:10.2337/ dc08-9026

Steven, S., Oelze, M., Hanf, A., Kröller-Schön, S., Kashani, F., Roohani, S., et al. (2017). The SGLT2 Inhibitor Empagliflozin Improves the Primary Diabetic Complications in ZDF Rats. Redox Biol. 13, 370-385. doi:10.1016/j.redox.2017. 06.009

Stevens, S. L., Wood, S., Koshiaris, C., Law, K., Glasziou, P., Stevens, R. J., et al. (2016). Blood Pressure Variability and Cardiovascular Disease: Systematic Review and Meta-Analysis. BMJ 354, i4098. doi:10.1136/bmj.i4098

Sun, Y. N., Zhou, Y., Chen, X., Che, W. S., and Leung, S. W. (2014). The Efficacy of Dapagliflozin Combined with Hypoglycaemic Drugs in Treating Type 2 Diabetes Mellitus: Meta-Analysis of Randomised Controlled Trials. BMJ Open 4 (4), e004619. doi:10.1136/bmjopen-2013-004619

Tan, Y., Yu, K., Liang, L., Liu, Y., Song, F., Ge, Q., et al. (2021). Sodium-glucose Cotransporter 2 Inhibition with Empagliflozin Improves Cardiac Function after Cardiac Arrest in Rats by Enhancing Mitochondrial Energy Metabolism. Front. Pharmacol. 12 (2820), 758080. doi:10.3389/fphar.2021.758080

Tang, W. H., Kitai, T., Hazen, S. L., and Hazen, S. L. (2017). Gut Microbiota in Cardiovascular Health and Disease. Circ. Res. 120 (7), 1183-1196. doi:10.1161/ CIRCRESAHA.117.309715

Tangvarasittichai, S. (2015). Oxidative Stress, Insulin Resistance, Dyslipidemia and Type 2 Diabetes Mellitus. World J. Diabetes 6 (3), 456-480. doi:10.4239/wjd.v6. i3.456

Thongnak, L., Pongchaidecha, A., Jaikumkao, K., Chatsudthipong, V., Chattipakorn, N., and Lungkaphin, A. (2017). The Additive Effects of Atorvastatin and Insulin on Renal Function and Renal Organic Anion Transporter 3 Function in Diabetic Rats. Sci. Rep. 7 (1), 13532. doi:10.1038/ s41598-017-13206-5

Thygesen, K., Alpert, J. S., Jaffe, A. S., Chaitman, B. R., Bax, J. J., Morrow, D. A., et al. (2018). Fourth Universal Definition of Myocardial Infarction (2018). Kardiol Pol. 76 (18), 1383-1415. doi:10.5603/KP.2018.0203

Tian, L., Cai, Y., Zheng, H., Ai, S., Zhou, M., Luo, Q., et al. (2021). Canagliflozin for Prevention of Cardiovascular and Renal Outcomes in Type2 Diabetes: a Systematic Review and Meta-Analysis of Randomized Controlled Trials. Front. Pharmacol. 12 (1658), 691878. doi:10.3389/fphar.2021.691878

Tietge, U. J. (2014). Hyperlipidemia and Cardiovascular Disease: Inflammation, Dyslipidemia, and Atherosclerosis. Curr. Opin. Lipidol. 25 (1), 94-95. doi:10. 1097/MOL.0000000000000051

Varbo, A., Benn, M., Tybjærg-Hansen, A., and Nordestgaard, B. G. (2013). Elevated Remnant Cholesterol Causes Both Low-Grade Inflammation and Ischemic Heart Disease, whereas Elevated Low-Density Lipoprotein Cholesterol Causes Ischemic Heart Disease without Inflammation. Circulation 128 (12), 1298-1309. doi:10.1161/CIRCULATIONAHA.113. 003008

Vikram, A., Kim, Y. R., Kumar, S., Li, Q., Kassan, M., Jacobs, J. S., et al. (2016). Vascular microRNA-204 Is Remotely Governed by the Microbiome and Impairs Endothelium-dependent Vasorelaxation by Downregulating Sirtuin1. Nat. Commun. 7, 12565. doi:10.1038/ncomms12565

Wang, L., Fouts, D. E., Stärkel, P., Hartmann, P., Chen, P., Llorente, C., et al. (2016). Intestinal REG3 Lectins Protect against Alcoholic Steatohepatitis by Reducing 
Mucosa-Associated Microbiota and Preventing Bacterial Translocation. Cell Host Microbe 19 (2), 227-239. doi:10.1016/j.chom.2016.01.003

Wang, X. L., Liu, D., Wang, Z., Cai, C., Jiang, H., and Yu, G. L. (2021). Porphyranderived Oligosaccharides Alleviate NAFLD and Related Cecal Microbiota Dysbiosis in Mice. FASEB J. 35 (6), e21458. doi:10.1096/fj.202000763rrr

Xiang, B., Zhao, X., and Zhou, X. (2021). Cardiovascular Benefits of SodiumGlucose Cotransporter 2 Inhibitors in Diabetic and Nondiabetic Patients. Cardiovasc. Diabetol. 20 (1), 78-14. doi:10.1186/s12933-021-01266-X

Yang, X. P., Lai, D., Zhong, X. Y., Shen, H. P., and Huang, Y. L. (2014). Efficacy and Safety of Canagliflozin in Subjects with Type 2 Diabetes: Systematic Review and MetaAnalysis. Eur. J. Clin. Pharmacol. 70 (10), 1149-1158. doi:10.1007/s00228-014-1730-x

Yayla, Ç., Gayretli Yayla, K., Açar, B., Unal, S., Ertem, A. G., Akboga, M. K., et al. (2017). White Blood Cell Subtypes and Ratios in Cardiovascular Disease. Angiology 68 (7), 651. doi:10.1177/0003319716664026

Zhang, J., Xia, L., Zhang, F., Zhu, D., Xin, C., Wang, H., et al. (2017). A Novel Mechanism of Diabetic Vascular Endothelial Dysfunction: HypoadiponectinemiaInduced NLRP3 Inflammasome Activation. Biochim. Biophys. Acta Mol. Basis Dis. 1863 (6), 1556-1567. doi:10.1016/j.bbadis.2017.02.012

Zheng, Y., Liu, T., Wang, Z., Xu, Y., Zhang, Q., and Luo, D. (2018). Low Molecular Weight Fucoidan Attenuates Liver Injury via SIRT1/AMPK/PGC1a axis in Db/db Mice. Int. J. Biol. Macromol 112, 929-936. doi:10.1016/j.ijbiomac.2018.02.072

Zhou, H., Wang, S., Zhu, P., Hu, S., Chen, Y., and Ren, J. (2018). Empagliflozin Rescues Diabetic Myocardial Microvascular Injury via AMPK-Mediated
Inhibition of Mitochondrial Fission. Redox Biol. 15, 335-346. doi:10.1016/j. redox.2017.12.019

Zinman, B., Lachin, J. M., and Inzucchi, S. E. (2016). Empagliflozin, Cardiovascular Outcomes, and Mortality in Type 2 Diabetes. N. Engl. J. Med. 374 (11), 1094. doi:10.1056/NEJMc1600827

Conflict of Interest: The authors declare that the research was conducted in the absence of any commercial or financial relationships that could be construed as a potential conflict of interest.

Publisher's Note: All claims expressed in this article are solely those of the authors and do not necessarily represent those of their affiliated organizations, or those of the publisher, the editors, and the reviewers. Any product that may be evaluated in this article, or claim that may be made by its manufacturer, is not guaranteed or endorsed by the publisher.

Copyright (C) 2022 Wang, Wang, Liu, Jiang, Cai, Li and Yu. This is an open-access article distributed under the terms of the Creative Commons Attribution License (CC $B Y)$. The use, distribution or reproduction in other forums is permitted, provided the original author(s) and the copyright owner(s) are credited and that the original publication in this journal is cited, in accordance with accepted academic practice. No use, distribution or reproduction is permitted which does not comply with these terms. 


\section{GLOSSARY}

T2DM type 2 diabetes mellitus

CVD cardiovascular disease

IR insulin resistance

Metf metformin

SGLT2i sodium glucose cotransporter 2 inhibitors

Cana canagliflozin

IPITT intraperitoneal insulin tolerance test

ROS reactive oxygen species;

OGTT oral glucose tolerance test

TC total cholesterol

TG triglycerides

HDL-C high-density lipoprotein cholesterol

LDL-C low-density lipoprotein cholesterol

PDW platelet distribution width

SOD superoxide dismutase

MPV mean platelet volume

AI atherogenic index

AIP atherogenic index of plasma

WBC white blood cell count

FBG fasting blood glucose
CD31 cluster of differentiation 31

MCV mean corpuscular volume

HbA1c hemoglobin Alc

RDW red cell distribution width

GSH glutathione

CAT catalase

MDA malondialdehyde

HOMA-IR homeostasis model assessment-insulin resistance

MCP-1 monocyte chemotactic protein 1

IL-6 interleukin-6

TNF-alpha tumor necrosis factor alpha

cTn I cardiac troponin I

SCD40L soluble cluster of differentiation 40 ligand

AMPK AMP-activated protein kinase

TEM transmission electron microscope

LefSe linear discriminate analysis effect size

SEM standard error of the mean

DHE dye dihydroethidium

SCFAs short-chain fatty acids

Dapa dapagliflozin

Empa empagliflozin 